\title{
Dispersion relation of triple-chambered cochlea
}

\author{
T. Kitamura \\ Faculty of Engineering Science, Kansai University, Osaka, 564-8680, Japan \\ Received: April 5, 2021. Revised: October 4, 2021. Accepted: October 20, 2021. Published: November 4, 2021.
}

\begin{abstract}
We investigated the dispersion relations of the triple-chambered cochlea separated by the basilar membrane and the Reissner's membrane. The slow waves can propagate on the basilar membrane (basilar membrane mode), and also on the Reissner's membrane (Reisnner's membrane mode). Most of the previous studies have focused on the mechanics of vibration of the basilar membrane and there are only a few studies on the wave propagation on Reissner's membrane. In this study, we focused particularly on the Reissner's membrane mode among the many propagation modes and evanescent modes that the structure supports. We analyzed the dispersion of the Reissner's membrane mode and the basilar membrane mode, and studied their dependency on the position along the cochlea. We also studied how the distance between the Reissner's membrane and the basilar membrane affects the dispersion relations.
\end{abstract}

Keywords-Reissner's membrane, basilar membrane, cochlea, mode analysis, dispersion relation, slow wave, wave propagation

\section{INTRODUCTION}

$\mathrm{T}$ HE cochlea is a fluid-filled duct that has three regions: the scala vestibuli, scala media, and scala tympani. They are separated by the Reissner's membrane (RM) and the basilar membrane (BM). Many analytical and numerical models have been developed to study wave propagation in the cochlea [1]. Most of the previous studies have treated the cochlea as two fluid chambers and focused on the mechanics of vibration of BM [2-9]. The slow wave that propagates on BM is called the BM mode $[10,11]$. On the other hand, there are only a few studies on the wave propagation on RM (RM mode). Fuhrmann et al. have studied the effects of RM on the wave propagation in the cochlea [12]. They assumed that RM had neither mass nor stiffness and treated it as a rigid boundary with no-slip condition. Rwichenbach et al. have shown that the RM mode plays an important role in transmitting the signals of otoacoustic emissions [13]. While BM includes radial fibers that impose a high stiffness, RM exhibits a comparatively low impedance. It has been shown that the RM mode does not evoke a significant displacement of BM, but, in contrast, a disturbance moving in the $\mathrm{BM}$ mode propagates on both membranes [13]. It has also been shown that in the basal region of the cochlea, and for frequencies above $1 \mathrm{kHz}, \mathrm{RM}$ sustains waves with wavelengths

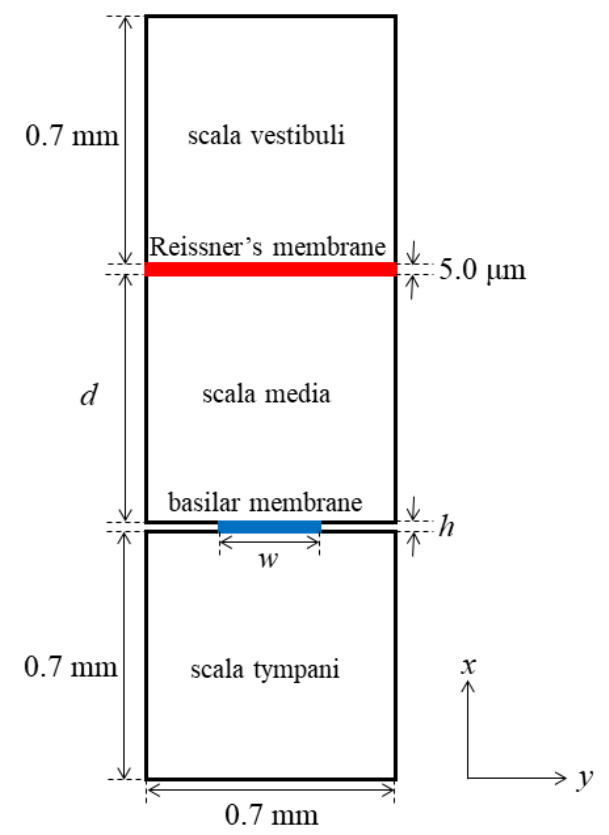

Figure 1. Cross-section of cochlea.

smaller than the height of the scalae and that do not penetrate into the membrane's surrounding fluids [13]. The dispersion relations of the RM mode, however, have not been studied so far. Given the dispersion relations, the phase velocity and group velocity can be obtained. Thereby, it is possible to understand the basic characteristics of the waves, such as fast wave and slow wave, and we can also discuss the possibility of the coupling between two modes.

In a previous study, we treated the cochlea as two fluid chambers and studied the dispersion diagrams of the fast-wave and slow-wave modes and the structural dependence of the angular wavenumber and coupling efficiency of the slow-wave mode [14]. We also investigated the dispersion characteristics of the propagation modes that progress on the tectorial membrane [15]. In this study, we considered the cochlea structure as three regions separated by RM and BM, and investigated the dispersion characteristics of the RM mode as 
well as the BM mode. We used COMSOL Multiphysics to analyze the effect of the elastic-wave mode.

\section{MODEL}

The human cochlea is a fluid-filled structure about $35 \mathrm{~mm}$ in length and coiled in a spiral of $21 / 2$ to $23 / 4$ turns. We modeled it as an uncoiled, triple-chambered fluid-filled duct, which is composed of the scala vestibuli, scala media, and scala tympani.

Figure 1 shows the analysis model. To simplify the problem, we assumed that RM and BM are located parallel to each other. Here, we also assumed that the cochlea duct was enclosed by rigid boundaries. The sizes of the three chambers; namely, the scala vestibuli, scala media, and scala tympani, vary along the cochlea duct. It has been shown in particular that the width of $\mathrm{RM}$ is slightly dependent on the location along the cochlea and has a length ranging from around 0.6 to $0.8 \mathrm{~mm} \mathrm{[16].} \mathrm{We}$ assumed here that the width of RM is constant at $0.7 \mathrm{~mm}$. The size of the chambers is uniform along the waveguide, and the heights and widths of the scala vestibuli and the scala tympani are equal at $0.7 \mathrm{~mm}$, which is the same length as the width of RM. We used the width $w$, height $h$, and Young's modulus $E$ of $\mathrm{BM}$ as parameters [17].

$$
\begin{gathered}
w=0.1[\mathrm{~mm}]+\frac{0.4[\mathrm{~mm}]}{35[\mathrm{~mm}]} \times z, \\
h=7.5[\mu \mathrm{m}]-\frac{5[\mu \mathrm{m}]}{35[\mathrm{~mm}]} \times z, \\
E=50[\mathrm{MPa}]-\frac{47[\mathrm{MPa}]}{35[\mathrm{~mm}]} \times z,
\end{gathered}
$$

where $z$ is the length along the cochlea duct. Here, the structural parameters of RM were assumed to be independent from the location along the cochlea. The length $d$ between RM and BM was used as a parameter, and we studied the influence of $d$ on the dispersion relations of the cochlea. The density and bulk modulus of the ideal fluid were respectively $1.034 \times 10^{3} \mathrm{~kg} / \mathrm{m}^{3}$ [18] and $2.2 \times 10^{9} \mathrm{~Pa}[1]$; the density and Poisson's ratio of $\mathrm{RM}$ and $\mathrm{BM}$ were respectively $1.2 \times 10^{3} \mathrm{~kg} / \mathrm{m}^{3}$ [18] and 0.49 [18]; and Young's modulus of RM was $15 \mathrm{MPa}$ [19]. We used COMSOL Multiphysics with 662 nodes and 8838 elements. In the analysis, the acoustic and elastic wave equations were solved by using the finite element method.

\section{MODE ANALYSIS}

Figure 2 shows the dispersion relation of the RM mode and the BM mode when $z=10,20$, and $30 \mathrm{~mm}$, where the horizontal axis is the acoustic-wave frequency $f$ and the vertical axis is the angular wavenumber $k$. Here, the length $d$ between RM and BM is $0.7 \mathrm{~mm}$ and the three chambers have the same square shape. As shown in this figure, the dispersion curves of the RM mode are almost the same regardless of $z$. On the other hand, those of the BM mode largely vary depending on $z$. When $z=10$ and 20 $\mathrm{mm}$, the RM mode has bigger angular wavenumbers compared with the BM mode over the whole frequency range, while they are smaller than those of the BM mode over the whole range when $z=30 \mathrm{~mm}$.

Figure 3 shows the $x$-direction displacement of the membrane of (a) RM mode and (b) BM mode when $f=1000$ $\mathrm{Hz}$, and (c) RM mode and (d) BM mode when $f=10000 \mathrm{~Hz}$, where $z=10 \mathrm{~mm}$. In these figures, the solid and dashed lines show the displacement of RM and BM, respectively. The vertical axis is normalized by the maximum value. As shown in Fig. 3(a), the RM mode vibrates only RM, while as in 3(b), the

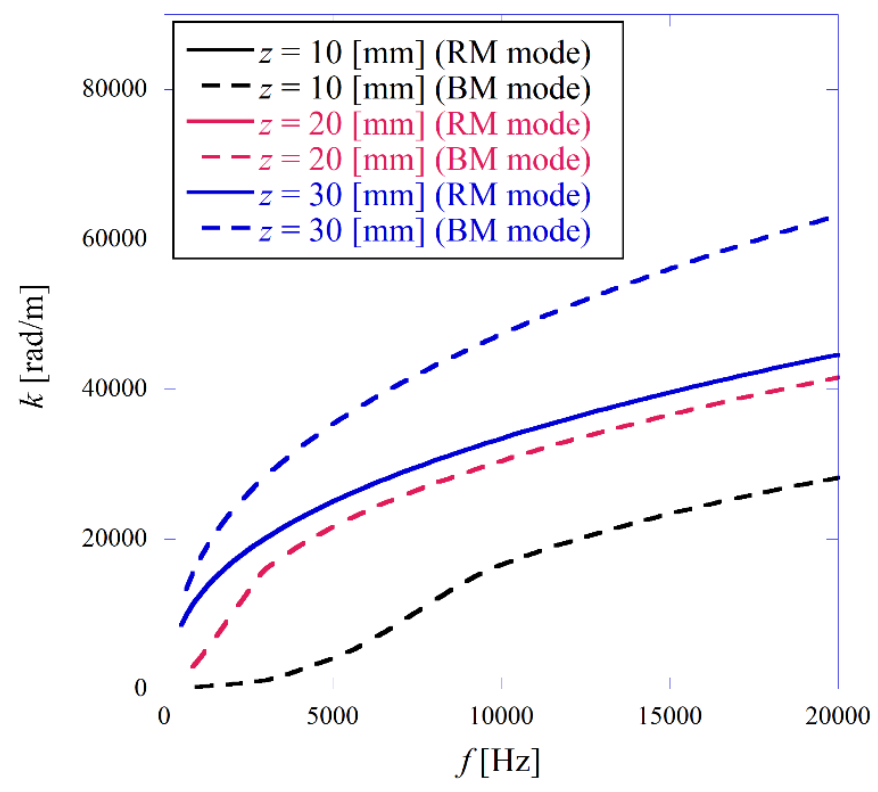

Figure 2. Dispersion diagram.

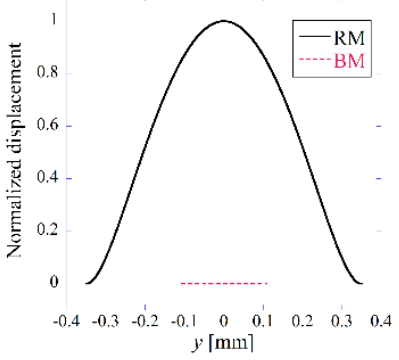

(a)

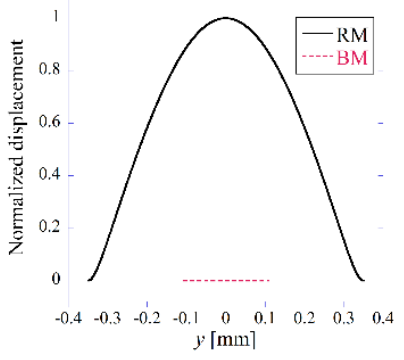

(c)

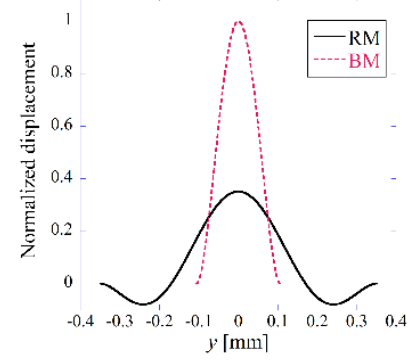

(b)

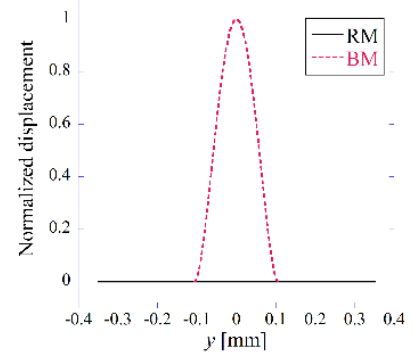

(d)
Figure 3. Displacement of membranes: (a) RM mode $(f=1000$ $\mathrm{Hz}$ ), (b) BM mode $(f=1000 \mathrm{~Hz}),(\mathrm{c}) \mathrm{RM}$ mode $(f=10000 \mathrm{~Hz})$, (d) BM mode $(f=10000 \mathrm{~Hz})$.

$\mathrm{BM}$ mode vibrates both $\mathrm{BM}$ and $\mathrm{RM}$ when $f=1000 \mathrm{~Hz}$. On the other hand, Fig. 3(c) and 3(d) show that each mode vibrates only its membrane when $f=10000 \mathrm{~Hz}$.

Figure 4 shows the angular wavenumber $k$ of the RM mode 
and the BM mode versus $z$, when $f=1000,3000$, and $10000 \mathrm{~Hz}$. This figure shows that the wavenumber of RM mode is constant from the base to the apex of the cochlea regardless of the acoustic frequency. On the other hand, the wavenumbers of the BM mode start to increase rapidly at a certain point along $z$. For example, when $f=10000 \mathrm{~Hz}$, the wavenumber has a small value

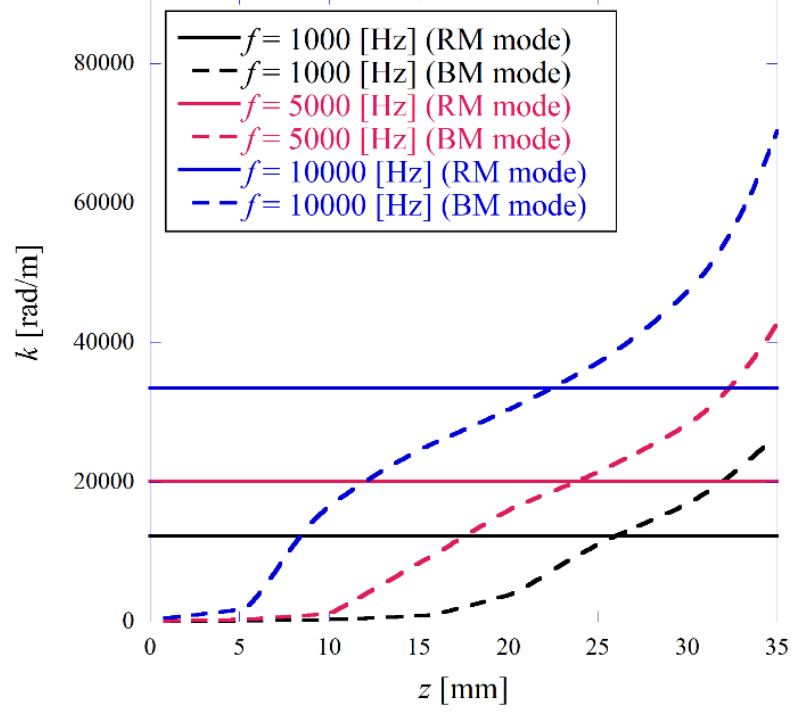

Figure 4. Angular wavenumber versus $z$.

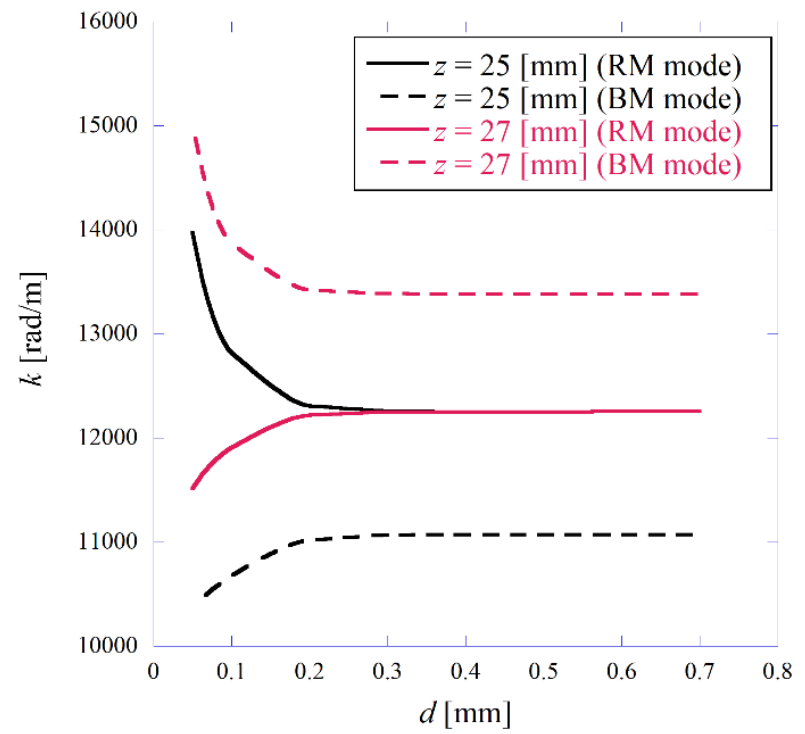

Figure 5. Angular wavenumber versus $d$ when $f=1000 \mathrm{~Hz}$.

until $z$ is less than about $5 \mathrm{~mm}$, and it starts to increase at that point. This is due to the variation along $z$ of the width, height, and Young's modulus of BM. We assumed that the Young's modulus of BM varies according to equation (3). Therefore, it is equal to that of RM when $z=26 \mathrm{~mm}$. We can see that the angular numbers of the two modes are equal when the Young's modulus of BM is slightly larger than that of RM.

Next, we investigated the influence of the height of the scala media $d$ on the angular wavenumber, wherein the heights of the other two chambers and the width of the three chambers are unchanged at $0.7 \mathrm{~mm}$. Fig. 5 shows the angular wavenumber $k$ of the RM mode and the BM mode versus $d$ when $f=1000 \mathrm{~Hz}$. It shows that $d$ hardly affects the angular wavenumbers of both modes when $d$ is more than $0.2 \mathrm{~mm}$. The differences of the angular wavenumbers between the two modes increase when $d$ is smaller than $0.2 \mathrm{~mm}$. The variation tendency, however, is not dependent on the mode type. For example, the angular

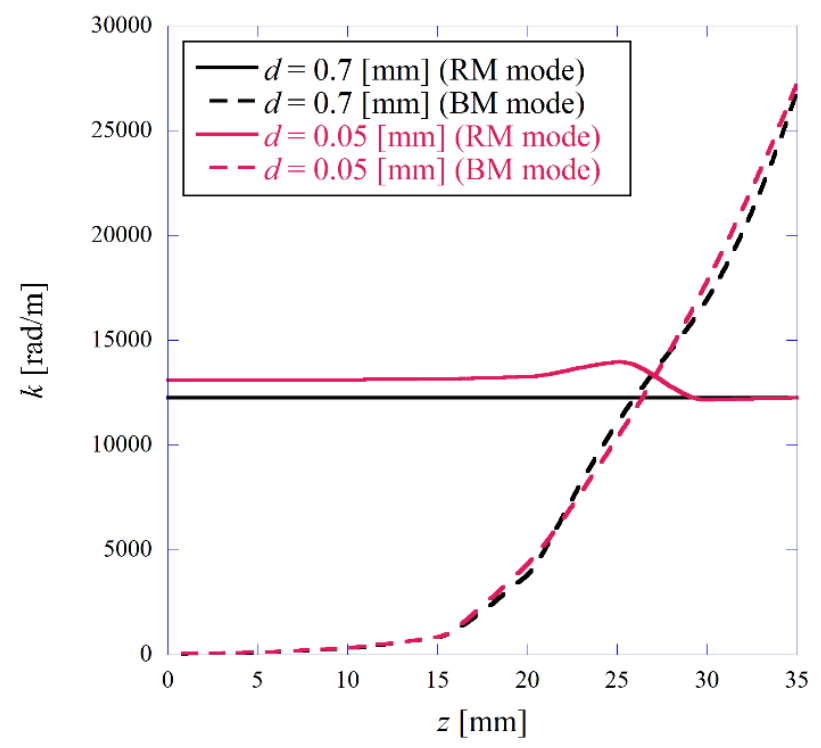

(a)

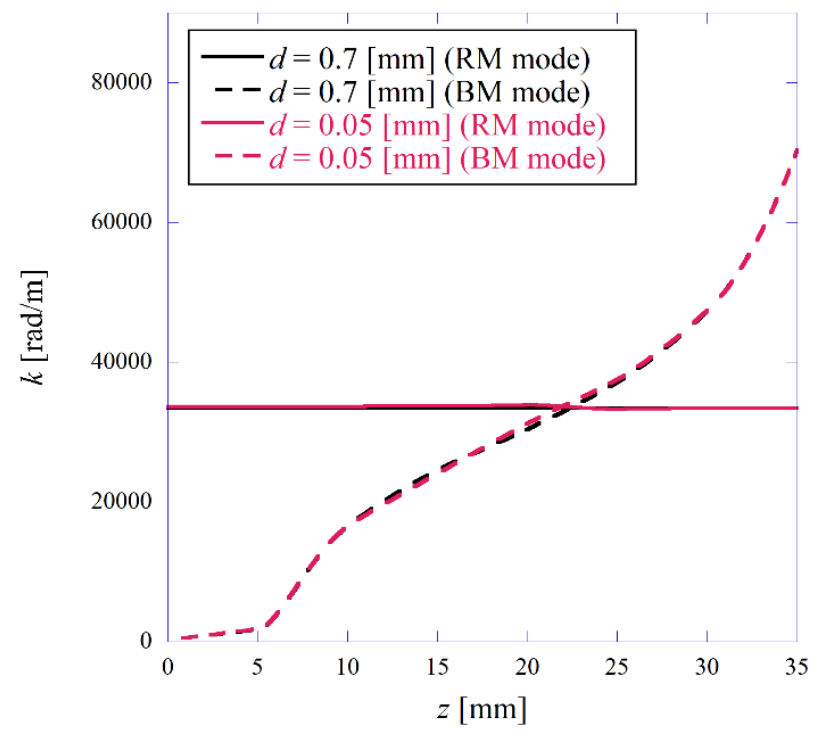

(b)

Figure 6. Angular wavenumber versus $z$ when (a) $f=1000$ $\mathrm{Hz}$ and (b) $f=10000 \mathrm{~Hz}$.

wavenumber of the RM mode increases as $d$ decreases when $z$ $=25 \mathrm{~mm}$, but it decreases when $z=27 \mathrm{~mm}$.

Figures 6(a) and 6(b) show the angular wavenumber $k$ of the RM mode and the BM mode versus $z$, when the height of the scala media $d$ is 0.05 or $0.7 \mathrm{~mm}$. Here, $f=$ (a) 1000 and (b) $10000 \mathrm{~Hz}$, respectively. As shown in Fig. 6(a), when $f=1000$ $\mathrm{Hz}$, the angular wavenumber $k$ of the RM mode increases as $d$ is reduced from 0.7 to $0.05 \mathrm{~mm}$. The angular wavenumber 
difference is about $1000 \mathrm{rad} / \mathrm{m}$ from $z=0$ to $25 \mathrm{~mm}$, and the difference becomes very small when $z$ is more than $30 \mathrm{~mm}$. On the other hand, $d$ has little influence on the angular wavenumber of the BM mode. From Fig. 6(b), we can see that both modes have little dependence on $d$ over the whole range of $z$ when $f=10000 \mathrm{~Hz}$

\section{CONCLUSION}

We investigated the cochlea, which has three regions separated by RM and BM, by using modal analysis. The dispersion diagrams of the RM mode and the BM mode showed that the wavenumber of the RM mode is constant from the base to the apex of the cochlea regardless of the acoustic frequency, while the wavenumber of the BM mode largely changes depending on the position along the cochlea. The analytical results of the displacement of the membranes demonstrated that when $f=1000 \mathrm{~Hz}$, the RM mode vibrates only RM, while the $\mathrm{BM}$ mode vibrates both $\mathrm{BM}$ and $\mathrm{RM}$, and that when $f=10000$ $\mathrm{Hz}$, each mode vibrates only its membrane. We also studied how the distance between BM and RM affects the dispersion relations of the $\mathrm{RM}$ mode and the $\mathrm{BM}$ mode. The results showed that when $f=1000 \mathrm{~Hz}$, the angular wavenumber of the $\mathrm{RM}$ mode increases as the distance is reduced, whereas when $f$ $=10000 \mathrm{~Hz}$, both modes have little dependence on the distance over the whole range of the length along the cochlea duct. It was shown that the distance hardly affects the angular wavenumbers of both modes when it is more than $0.2 \mathrm{~mm}$.

\section{REFERENCES}

[1] A. De Paolis, M. Bikson, J. T. Nelson, J. A. de Ru, M. Packer, and L. Cardoso, "Analytical and numerical modeling of the hearing system: advances towards the assessment of hearing damage," Hear. Res., vol. 349, pp. 111-128, 2017.

[2] J. B. Allen, "Two-dimensional cochlear fluid model: new results," J. Acoust. Soc. Am., vol. 61, no. 1, pp.110-119, Jan. 1977.

[3] C. R. Steele and L. A. Taber, "Comparison of WKB calculations and experimental results for three-dimensional cochlear models," J. Acoust. Soc. Am., vol. 65, no. 4, pp.1007-1018, Apr. 1979.

[4] S. T. Neely, "Finite difference solution of a two-dimensional mathematical model of the cochlea," J. Acoust. Soc. Am., vol. 69, no. 5, pp. 1386-1393, May 1981.

[5] E. de Boer and E. van Bienema, "Solving cochlear mechanics problems with higher-order differential equations," J. Acoust. Soc. Am., vol. 72, no. 5, pp. 1427-1434, Nov. 1982.

[6] M. H. Holmes, "Frequency discrimination in the mammalian cochlea: theory versus experiment," J. Acoust. Soc. Am., vol 81, no. 1, pp. 103-114, Jan. 1987.

[7] A. A. Parthasarathi, K. Grosh, and A. L. Nuttall, "Three-dimensional numerical modeling for global cochlear dynamics," J. Acoust. Soc. Am., vol. 107, no. 1, pp. 474-485, Jan. 2000.
[8] G. Ni and S. J. Elliot, "Comparing methods of modeling near field fluid coupling in the cochlea," J. Acoust. Soc. Am., vol. 137, no. 3, pp. 1309-1317, Mar. 2015.

[9] E. Edom, D. Obrist, R. Henniger, and L. Kleiser, "The effect of rocking stapes motions on the cochlear fluid flow and on the basilar membrane motion," J. Acoust. Soc. Am. vol. 134, no. 5, pp. 3749-3758, Nov. 2013.

[10] J. Lighthill, "Energy flow in the cochlea," J. Fluid Mech., vol. 106, pp. 149-213, 1981.

[11] M. Andoh and H. Wada, "Prediction of the characteristics of two types of pressure waves in the cochlea: theoretical considerations," J. Acoust. Soc. Am., vol. 116, no. 1, pp. 417-425, July 2004.

[12] E. Fuhrmann, W. Schneider, and M. Schultz, "Wave propagation in the cochlea (inner ear): effects of Reissner's membrane and non-rectangular cross-section," Acta Mechanica, vol. 70, pp. 15-30, 1987.

[13] T. Reichenbach, A. Stefanovic, F. Nin, and A. J. Hudspeth, "Waves on Reissner's membrane: a mechanism for the propagation of otoacoustic emissions from the cochlea," Cell Reports, vol. 1, no. 4, pp. 374-384. Apr. 2012.

[14] T. Kitamura, "Investigation of coupling efficiency of slow-wave propagation mode along cochlea," Phys. Wave Phenom., vol. 27, no.3, pp. 242-245, 2019.

[15] T. Kitamura, "Mode analysis of tectorial membrane in cochlea," Biomed. Pharmacol. J., vol. 14, no.3, pp. 1389-1395, 2021.

[16] C. R. Steele, “Stiffness of Reissner's membrane," J. Acoust. Soc. Am., vol. 56, no. 4, pp. 1252-1257, Oct. 1974.

[17] R. Z. Gan, B. P. Reeves, and X. Wang, "Modeling of sound transmission from ear canal to cochlea," Ann. Biomed. Eng. vol. 35, no. 12, pp. 2180-2195, Dec. 2007.

[18] T. Koike, C. Sakamoto, T. Sakashita, K. Hayashi, S. Kanzaki, and K. Ogawa, "Effects of a perilymphatic fistula on the passive vibration response of the basilar membrane," Hear. Res., vol. 283, pp. 117-125, 2012.

[19] W. Yao Y. Chen, J. Ma, C. Gan, and D. Wang, "Numerical simulation on the dynamic behavior of the basilar membrane in the spiral cochlea," Biomedical Research vol. 27, no. 3, pp. 977-984, 2016.

\section{Creative Commons Attribution License $\mathbf{4 . 0}$ (Attribution 4.0 International, CC BY 4.0)}

This article is published under the terms of the Creative Commons Attribution License 4.0 https://creativecommons.org/licenses/by/4.0/deed.en_US 